Aus dem Anatomischen Institut der Med. Fakultät Okayama

(Vorstand : Prof. M. SEKI).

\title{
Vergleichende histometrische Studien über die Netzhaut der Wirbeltiere.
}

脊椎動物の網膜の比較組織测定的研究.

Goro SUDO. 須藤 五 郎.

(Eingegangen 28. Oktober 1949.)

Es wurde früher (1950) die Dicke der Netzhautschichten der entwickelnden Hühner gemessen, und die folgenden Tatsachen wurden beobachtet: Die relative Dicke der Zapfenstäbchenschicht bezogen auf die ganze Retinadicke vermehrt sich bis zur Geburt, aber nachher vermehrt sie nicht. Diejenigen der inneren und der äußeren Körnerschicht vermindern sich mit dem Wachstum. Demgegenüber vermehrt die innere retikuläre Schicht ihre relative Dicke. Der Kernteil der bjpolaren Zellen nähert sich während der Entwicklung den Sehzellen, also.das Gesetz von KAPPERS der sog. Neurobiotaxis ist auch hier gültig. Ich habe nun neuerdings dieselben Untersuchungen bei verschiedenen Arten erwachsener Wirbeltiere angestellt.

\section{Material und Methode.}

Fische : Aal und Makrele, Amphibien : Salamander, Bufo vulgaris und Rana nigromaculata, Reptilien : Schlange, Takydromus tachydromoides, Eumeces latisculatus und schildkröte, Vögel : Huhn, Säugetiere : Fledermaus, Kaninchen und Mensch, all diese standen mir als Versuchsgegenstände zur Verfügung. Ich fixierte die Augäpfel dieser Tiere in Formalinlösung, stellte $15 \mu$ dicke Celloidinschnitte her und färbte diese mit Hämatoxylin und Eosin. Die Netzhautschichten wurden am hinteren Teil des Augapfels, Fovea bzw. Area centralis vermieden, durch den Okularmikrometer gemessen.

\section{Ergebnisse der Messung.}

1. Der absolute Wert der Dicke der Netzhautschichten ist in den Tabellen 1-5, der Bau der Netzhaut in der Tafelabbildung gezeigt.

2'. Aus der Tab. 6 und der Abb. 1 ergibt sich bezüglich der relativen Dicke der äußeren Körnerschicht die Reihenfolge : Fische= Säugetiere $>$ Amphibien $>$ Reptilien $>$ Vogel. Nach SCHULTZE (1867) sind in der Zapfenstäbchenschicht der Knochenfische mehr Siäbchen 
Tabelle 1. Dicke der Netzhautschichten der Fische.

\begin{tabular}{|c|c|c|c|c|}
\hline & \multicolumn{2}{|l|}{ Aal } & \multicolumn{2}{|c|}{ Makrele } \\
\hline & $\mathbf{M} \pm \mathbf{m}$ & $\mathrm{V}$ & $\mathrm{M} \pm \mathrm{m}$ & $\mathrm{V}$ \\
\hline Zapfenstäbchenschicht & $15.97 \pm 0.18$ & 10.25 & $23.25 \pm 0.24$ & 10.64 \\
\hline äu ßere Körnerschicht & $27.20 \pm 0.25$ & 9.28 & $68.26 \pm 0.26$ & 4.15 \\
\hline HENIEsche Schicht & 0 & & $10.45 \pm 0.15$ & 14.93 \\
\hline äußere retikuläre Schicht & $14.76 \pm 0.36$ & 24.47 & $4.40 \pm 0.12$ & 28.40 \\
\hline innere Körnerschicht & $20.23 \pm 0.21$ & 10.62 & $53.80 \pm 0.53$ & 10.15 \\
\hline innere retikuläre Schicht & $32.77 \pm 0.22$ & 6.78 & $46.12^{\circ} \pm 0.67$ & 12.00 \\
\hline Ganglienzellenschicht & $3.01 \pm 0.10$ & 14.09 & $14.32 \pm 0.20$ & 14.57 \\
\hline Nervenfaserschicht & $2.01 \pm 0.08$ & 41.61 & $185.65 \pm 1.24$ & 6.76 \\
\hline Netzhaut & $115.95 \pm 0.18$ & 1.63 & $406.25 \pm 0.36$ & 0.91 \\
\hline
\end{tabular}

Tabelle 2. Dicke der

\begin{tabular}{l|c|c|}
\hline & \multicolumn{2}{|c|}{ Salamander } \\
\cline { 2 - 3 } & $\mathrm{M} \pm \mathrm{m}$ & $\mathrm{V}$ \\
\hline Zapfenstäbchenschicht & $7.28 \pm 0.08$ & 13.12 \\
äußere Körnerschicht & $23.95 \pm 0.21$ & 9.08 \\
HENLEsche Schicht & 0 & \\
äußere retikuläre Schicht & $3.01 \pm 0.15$ & 51.45 \\
innere Körnerschicht & $69.05 \pm 0.46$ & 6.75 \\
innere retikuläre Schicht & $28.12 \pm 0.25$ & 8.88 \\
Ganglienzellenschicht & $37.01 \pm 0.20$ & 5.63 \\
Nervenfaserschicht & $12.12 \pm 0.28$ & 8.88 \\
Netzhaut & $180.54 \pm 0.24$ & 1.36
\end{tabular}

Tabelle 3. Dicke der

\begin{tabular}{|c|c|c|c|}
\hline & \multicolumn{2}{|c|}{ Schlange } & $\begin{array}{c}\text { Takydromus } \\
\text { tachydromoides }\end{array}$ \\
\hline & $\mathrm{M} \pm \mathrm{m}$ & V & $M \pm \mathrm{m}$ \\
\hline Zapfenstäbchenschicht & $12.11 \pm 0.06$ & 5.46 & $17.97 \pm 0.13$ \\
\hline äußere Körnerschicht & $12.53 \pm 0.10$ & 8.10 & $17.47 \pm 0.11$ \\
\hline HENIEsche Schicht & $5.18 \pm 0.09$ & 18.79 & $5.91 \pm \mathbf{0 . 0 6}$ \\
\hline äußere retikuläre $S \operatorname{chich}$ & $6.86 \pm 0.08$ & 12.38 & $1.35 \pm 0.05$ \\
\hline innere Körnerschicht & $43.91 \pm 0.30$ & 6.88 & $64.97 \pm 0.30$ \\
\hline innere retikuläre Schicht & $29.12 \pm 0.19$ & 6.65 & $62.70 \pm 0.41$ \\
\hline Ganglienzellenschicht & $8.93 \pm 0.24$ & 26.99 & $15.35 \pm 0.13$ \\
\hline Nervenfaserschicht & $13.17 \pm 0.17$ & 12.09 & $17.12 \pm 0.22$ \\
\hline Netzhaut & $131.81 \pm 0.29$ & 2.51 & $202.84 \pm 0.20$ \\
\hline
\end{tabular}


Vergleichende histometrische Studien über d. Netzhaut usw.

Tabelle 4. Dicke der Netzhautschichten des Huhns.

\begin{tabular}{l|r|r}
\hline & \multicolumn{2}{|c}{ Huhn } \\
\cline { 2 - 3 } & $\mathrm{M} \pm \mathrm{m}$ & $\mathrm{V}$ \\
\hline Zapfenstäbchenschicht & $\mathbf{1 6 . 1 9 \pm 0 . 2 1}$ & 13.55 \\
äußere Körnerschicht & $6.18 \pm 0.07$ & 12.87 \\
HENLEsche Schicht & $1.23 \pm 0.01$ & 15.40 \\
äußere retikuläre Schicht & $3.30 \pm 0.05$ & 17.51 \\
innere Körnerschicht & $13.21 \pm 0.24$ & 18.36 \\
innere retikuläre Schicht & $57.47 \pm 0.38$ & 6.64 \\
Ganglienzellenschicht & $9.36 \pm 0.15$ & $\mathbf{1 7 . 1 3}$ \\
Nervenfaserschicht & $79.17 \pm 1.01$ & 13.10 \\
Netzhaut & $185.45 \pm 0.38$ & 2.09
\end{tabular}

Netzhautschichten der Amphibien.

\begin{tabular}{|c|c|c|c|}
\hline \multicolumn{2}{|c|}{ Bufo vulgaris } & \multicolumn{2}{|c|}{ Rana nigromaculata } \\
\hline $\mathbf{M} \pm \mathrm{m}$ & V & $\mathbf{M} \pm \mathbf{m}$ & $\mathrm{V}$ \\
\hline $12.75 \pm 0.16$ & 12.54 & $2.63 \pm 0.06$ & 25.59 \\
\hline $22.21 \pm 0.15$ & 6.92 & $13.61 \pm 0.11$ & 8.35 \\
\hline $2.31 \pm 0.06$ & 24.34 & $3.60 \pm 0.93$ & 26.114 \\
\hline $4.21 \pm 0.09$ & 23.44 & $1.97 \pm 0.09$ & 46.83 \\
\hline $45.26 \pm 0.37$ & 8.11 & $33.26 \pm 0.14$ & 4.28 \\
\hline $46.55 \pm 0.19$ & 4.21 & $31.22 \pm 0.96$ & 31.02 \\
\hline $7.95 \pm 0.10$ & 13.05 & $6.70 \pm 0.07$ & 10.82 \\
\hline $9.63 \pm 0.13$ & 14.13 & $16.20 \pm 0.23$ & 14.35 \\
\hline $150.37 \pm 0.46$ & 3.12 & $109.19 \pm 0.57$ & 5.24 \\
\hline
\end{tabular}

Netzhautschichten der Reptilien.

\begin{tabular}{|c|c|c|c|c|}
\hline \multirow[b]{2}{*}{$\mathrm{V}$} & \multicolumn{2}{|c|}{ Eumeces latisculatus } & \multicolumn{2}{|c|}{ Schildkröte } \\
\hline & $\mathrm{M} \pm \mathrm{m}$ & $\mathrm{V}$ & $\mathbf{M} \pm \mathrm{m}$ & $\mathrm{v}$ \\
\hline 7.16 & $16.17 \pm 0.17$ & 10.59 & $24.77 \pm 0.11$ & 4.49 \\
\hline 6.58 & $17.43 \pm 0.08$ & 50.82 & $14.75 \pm 0.14$ & 9.58 \\
\hline 11.41 & $2.45 \pm 0.05$ & 21.87 & $2.53 \pm 0.08$ & 32.50 \\
\hline 27.03 & $4.26 \pm 0.06$ & 14.07 & $3.77 \pm 0.07$ & 18.87 \\
\hline 4.65 & $36.82 \pm \mathbf{0 . 0 8}$ & 2.30 & $28.37 \pm 0.20$ & 7.22 \\
\hline 6.54 & $51.46 \pm 0.15$ & 2.91 & $49.67 \pm 0.14$ & 2.96 \\
\hline 8.55 & $8.94 \pm 0.15$ & 16.99 & $9.56 \pm 0 \cdot 17$ & 17.77 \\
\hline 13.35 & $15.11 \pm 0.13$ & 8.91 & $19.15 \pm 0.10$ & $5 \cdot 48$ \\
\hline 1.02 & $152.64 \pm 0.37$ & 2.48 & $152.57 \pm 0.47$ & 3.10 \\
\hline
\end{tabular}


Tabelle 5..' Dicke der

\begin{tabular}{l|c|c|}
\hline & \multicolumn{2}{|c|}{ Fledermaus } \\
\cline { 2 - 3 } & $\mathrm{M} \pm \mathrm{m}$ & $\mathrm{V}$ \\
\hline Zapfenstäbchenschicht & $23.96 \pm 0.16$ & 6.77 \\
äußere Körnerschicht & $22.90 \pm 0.09$ & 4.03 \\
HENLEsche Schicht & 0 & \\
äußere retikuläre Schicht & $4.95 \pm 0.08$ & 17.92 \\
innere Körnerschicht & $17.92 \pm 0.06$ & 3.48 \\
innere retikuläre Schicht & $16.85 \pm 0.17$ & 10.46 \\
Ganglienzellenschicht & $7.38 \pm 0.09$ & 12.52 \\
Nervenfaserschicht & $2.55 \pm 0.07$ & 28.00 \\
Netzhaut & $96.5 \pm 0.87$ & 9.12
\end{tabular}

Tabelle 6. Relative

\begin{tabular}{l|c|c|c|c|r|}
\hline & \multicolumn{2}{|c|}{ Fische } & \multicolumn{3}{c|}{ Amphibien } \\
\cline { 2 - 6 } & Aal & Makrele & $\begin{array}{c}\text { Sala- } \\
\text { mander }\end{array}$ & $\begin{array}{c}\text { Bufo } \\
\text { vulg. }\end{array}$ & $\begin{array}{c}\text { Rana } \\
\text { nigrom. }\end{array}$ \\
\hline Zapfenstäbchenschicht & 13.88 & 5.72 & 4.04 & 8.50 & 2.41 \\
äßere Körnerschicht & 23.65 & 16.81 & 13.30 & 14.80 & 12.48 \\
HENLEsche Schicht & 0 & 2.57 & 0 & 1.54 & 3.30 \\
äßere retikuläre Schicht & 12.83 & 1.08 & 1.60 & 2.80 & 1.80 \\
innere Körnerschicht & 17.59 & 13.25 & 38.36 & 30.16 & 30.51 \\
innere retikuläre Schicht & 28.48 & 11.35 & 15.61 & 31.03 & 28.64 \\
Ganglienzellenschicht & 2.61 & 3.52 & 20.56 & 5.30 & 6.14 \\
Nervenfaserschicht & 1.74 & 45.71 & 6.73 & 6.42 & $\mathbf{1 4 . 8 6}$ \\
\hline
\end{tabular}

vorhanden, bei den Tiefseefischen und dem Aal besteht die Schicht sogar nur aus den Stäbchen. Bei dem Salamander, dem Bufo vulgaris und der Rana nigromaculala entwickeln sich die Słäbchen, worauf FRANZ (1913) aufmerksam gemacht hat, sehr gut. Nach KRAUSE (1893 u. 1895) besteht die Zapfenstäbchenschicht bei der Fledermaus und dem Kaninchen fast nur aus den Stäbchen. Bei der Schlange und dem Eumeces latisculatus sind die Stäbchen dagegen spärlich, und der Schildkröte fehlen die Stäbchen gänzlich.

Nach SCHULTZE (1866) sind bei Nachtvögeln mehr Stäbchen, bei den Tagvögeln aber weniger in der Retina zu sehen. Es isi also anzunehmen, daß die relative Dicke der äußeren Körnerschicht bei solchen Wirbeltieren, die an den Stäbchenzellen reich sind, groß ist, daß sie aber bei solchen, die an den Zapfenzellen reich sind, kleiner ist. 
Netzhautschichten der Säugetiere.

\begin{tabular}{|c|c|c|c}
\hline \multicolumn{2}{c|}{ Kaninchen } & \multicolumn{2}{c}{ Mensch } \\
\hline $\mathrm{M} \pm \mathrm{m}$ & $\mathrm{V}$ & $\mathrm{M} \pm \mathrm{m}$ & $\mathrm{V}$ \\
\hline $16.05 \pm \mathbf{0 . 1 8}$ & 11.68 & $49.31 \pm 0.23$ & 4.84 \\
$16.87 \pm \mathbf{0 . 1 9}$ & $1 \mathrm{I} .77$ & $46.08 \pm 0.21$ & 4.74 \\
$\mathbf{2 . 0 3} \pm 0.06$ & 29.44 & $4.80 \pm 0.10$ & 22.39 \\
$2.87 \pm 0.08$ & 30.86 & $7.23 \pm 0.12$ & 16.58 \\
$11.87 \pm 0.08$ & 6.73 & $26.10 \pm 0.20$ & 7.71 \\
$29.68 \pm 0.23$ & 7.83 & $24.91 \pm 0.19$ & 7.97 \\
$10.57 \pm 0.16$ & 15.48 & $19.33 \pm \mathbf{0 . 2 1}$ & 10.85 \\
$5.63 \pm 0.10$ & 18.62 & $24.26 \pm \mathbf{0 . 2 4}$ & 10.20 \\
$95.57 \pm 0.67$ & 7.11 & $202.02 \pm 0.22$ & 1.11 \\
& & &
\end{tabular}

Dicke der Netzhautschichten.

\begin{tabular}{|c|r|r|r|r|r|r|r}
\hline \multicolumn{3}{|c|}{ Reptilien } & Vögel & \multicolumn{3}{|c}{ Säugetiere } \\
\cline { 1 - 4 } Schlang e & $\begin{array}{c}\text { Taky- } \\
\text { dromus }\end{array}$ & Eumeces & $\begin{array}{c}\text { Schild } \\
\text { kröte }\end{array}$ & Huhn & $\begin{array}{c}\text { Fleter- } \\
\text { maus }\end{array}$ & $\begin{array}{c}\text { Kanin- } \\
\text { chen }\end{array}$ & Mensch \\
\hline 9.24 & 8.89 & 10.63 & 16.29 & 8.75 & 24.95 & 16.39 & 24.41 \\
9.56 & 8.64 & 11.46 & 9.70 & 3.34 & 23.85 & 17.75 & 22.81 \\
3.95 & 2.92 & 1.61 & 1.66 & 0.66 & 0 & 2.13 & 2.37 \\
5.23 & 0.66 & 2.80 & 2.48 & 1.78 & 5.15 & 3.02 & 3.57 \\
33.51 & 32.16 & 24.22 & 18.66 & 7.14 & 18.66 & 12.49 & 12.92 \\
22.22 & 31.03 & 33.35 & 32.67 & 31.06 & 17.55 & 31.24 & 12.33 \\
6.81 & 7.59 & 5.88 & 6.23 & 5.05 & 7.68 & 11.12 & 9.56 \\
10.05 & 8.47 & 9.94 & 12.59 & 42.25 & 2.61 & 5.92 & 12.00 \\
& & & & & &
\end{tabular}

Die relative Dicke der inneren Körnerschicht ist bei Amphibien> Reptilien $>$ Säugetieren $>$ Fischen $>$ Vögel. Die, relative Dicke der äußeren Körnerschicht ist bei den Fjschen und Süugetieren größer als diejenige der inneren Körnerschicht, aber bei den Amphibien, Reptilien und Vögeln ist das Umgekehrte der Fall.

3. Bei denselben Arten von Tieren ist die relative Dicke der Zapfenstäbchenschicht bezogen auf die ganze Retinadicke, wie man es aus der Tab. 6 und der Abb. 2 ersieht, bei den Fischen : Aal> Makrele, bei den Amphibien: Bufo vulgaris $>$ Salamander $>$ Rana nigromaculata, bei den Reptilien : Eumeces latisculatus $>$ Schlange $>$ Takydromus tachydromoides und bei den Säugetieren : Fledermaus $>$ Mensch>Kaninchen. Die relative Dicke der äußeren Körnerschicht verhält sich gleich. Kurz, wenn bei denselben Arten Tieren die 
relative Dicke der Zapfenstäbchenschicht groß ist, so ist diejenige der äußeren Körnerschicht auch groß. $Z_{w}$ ischen beiden schichten ist eine intime Verbindung zu finden.

Die HENLEsche Faserschicht jst unter den Fischen, beim Aal nicht, bei der Makrele aber gut entwickelt. Bei den Amphibien findet man sie nucht beim Salamander, wohl aber bei den anderen beiden Tieren, und die relative Dicke : Rana nigromaculata $>$ Bufo vulgaris. Bei den Säugetieren fehlt sie bei der Fledermaus, bej den anderen bestätigt man die relative Dicke : Mensch $>$ Kaninchen. Unter den Tieren derselben Art ist die HENLEsche Faserschicht bej solchen besser entwickelt, die im helleren Ort leben. Diese Tatsache stimmt damit gut überein, $d a B$ in der Fovea centralis retinae des Menschen, welche für das Licht aufs beste empfindlich ist, die HENLEsche Faserschicht am besten entwickelt ist. Bei den

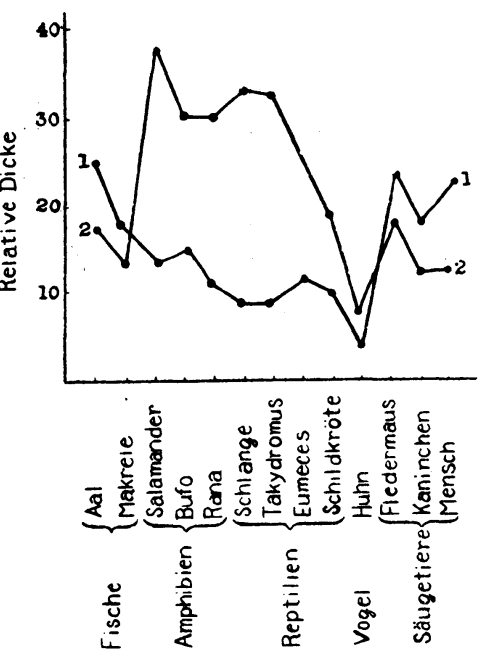

Abb. 1. Relative Dicke der äußeren und inneren Körnerschicht der Wirbeltiernetzhaut. 1. äußere Körnerschicht, 2. innere Körnerschicht. Reptilien wird folgende Reihenfolge der relativen Dicke gefunden : Schlange $>$ Takydromus tachydromoides $>$ Eumeces latisculatus $>$ Sichildkröte. Beim Huhn ist aber dje Entwicklung der Schicht schlecht.

Die relative Dicke der äußeren retikulären Schicht ist fast jmmer klein, abgesehen vom Aal, wo sie groß ist.

Die relative Dicke der inneren Körnerschicht (s. Tab. 6 und Abb. 2) ist bei den Fischen : Aal $>$ Makrele, bei den Amphibien : Salamander $>$ Bufo vulgaris = Rana nigromaculata, bei den Reptilien : Schlange $>$ Takydromus tachydromoldes $>$ Eumeces latisculatus $>$ Schildkröte und bei den Säugetjeren : Fledermaus $>$ Kaninchen $>$ Mensch. Die relative Dicke ist also bei den Tieren derselben Art umso kleıner, je größer der Augapfel ist.

Dies scheint dadurch verursacht zu sein, daß bei den Tieren mit großem Augapfel die innere Körnerschicht sich mehr flach breitet.

In der Ganglienzellenschicht reihen sich die Zellen einschichtig, nur bei dem Salamander häufen sie sich auf 3 bis 4 Schichten. 
Die relative Dicke der Nervenfaserschicht (s. Tab. 6 und Abb. 2) ist bei den Fischen : Makrele>Aal, bei den Amphibien : Rana nigromacula1a $>$ Bufo vulgaris = Salamander, bej den Reptilien : Schildkröte

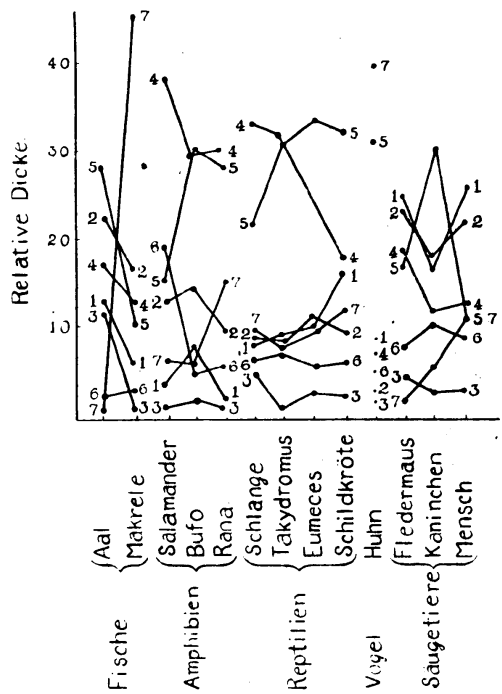

Abb. 2. Relative Dicke der Netzhautschichten. 1. Zapfenstäbchenschicht, 2. äußere Körnerschicht, 3. äußere retikuläre Schicht, 4. innere Körnerschicht, 5. innere retikuläre Schicht, 6 . Ganglienzellenschicht, 7. Nervenfaserschicht.
$>$ Eumeces latisculatus = Schlange $>$ Takydromus 1achydromoides, bei den Säugetieren : Mensch $>\mathrm{Ka}$ ninchen $>$ Fledermaus. Man kann sagen, daß unter den Tieren einer Art die relative Dicke der Nervenfaserschicht umso größer ist, je besser die Sehkraft ist.

4. Der Kernteil der bipolaren Zellen nimmt auf der verschiedenen Höhe der inneren Körnerschicht Platz. Der Dendrit ende1 auf die äußerste Zone der äußeren retikulären Schjcht, der Neurit auf die verschiedene Höhe der inneren retikulären Schicht. Die durchschnittliche Lage des Kernteils ist in der Kritte der inneren Körnerschicht zu finden. Die Lage des Endpunktes des Neuriten scheint durchschnittlich in der Mitte der inneren retikulären Schicht zu finden. Folglich ist die L̈̈̈nge des Dendriten durchschnittlich die halbe Dicke der inneren Körnerschicht, plus die Dicke der äußeren retikulären schicht. Und die

Iänge des Neuriten ist durchschnittlich die halbe Dicke der inneren Körnerschicht plus die halbe Dicke der inneren retikulären Schicht. Das Verhältnis : Länge des Dendriten $\times 100 /$ Länge des Neuriten, bezeichnet man als der Dendritenindex der bipolaren Zellen. Der Dendritenindex der Netzhaut (Abb.3) ist : Aal > Makrele, Salamander $>$ Bufo vulgaris $>$ Rana nigromaculata, Schlange $>$ Takydromus tachydromoides $>$ Eumeces latisculatus $>$ Schildkrote, Flcdermaus $>$ Mensch $>$ Kaninchen $<$ Huhn.

Der Aal lebt unter dem Wasser im dunkeln und erhält sehr wenig Licht, dagegen schwimmt die Makrele in belleren, lichtreichen Teilen der See. Der Salamander lebt meistens im dunklen Wassersgrund. Der Bufo vulgaris hat dunkle Orte gern, aber die Rana nigromaculata 
lebt lieber im hellen. Die Schlange bleibt stets im Dunkeln, aber der Takydromus tachydromoides und Eumeces latisculatus sind meistens unter dem starken sonnenlicht tätig und erhalten weit mehr Licht als die Schlange. Auch die Schildkröte pflegt sich im Sonnenschein zu Jegen, wenn niemand in der Nähe ist; sje scheint im hellen Ort gern zu leben. Die Fledermaus fliegt gern in der Nacht. Der Mensch und das Kaninchen leben im hellen. Kurz, unter den Tieren derselben Art erweist sich der Dendritenindex der bipolaren Zellen bei dem Tiere, welches im helleren wohnt, meistens als kleiner. Hier gilt auch das KAPPERSsche Gesetz der sog. Neurobiotaxis.

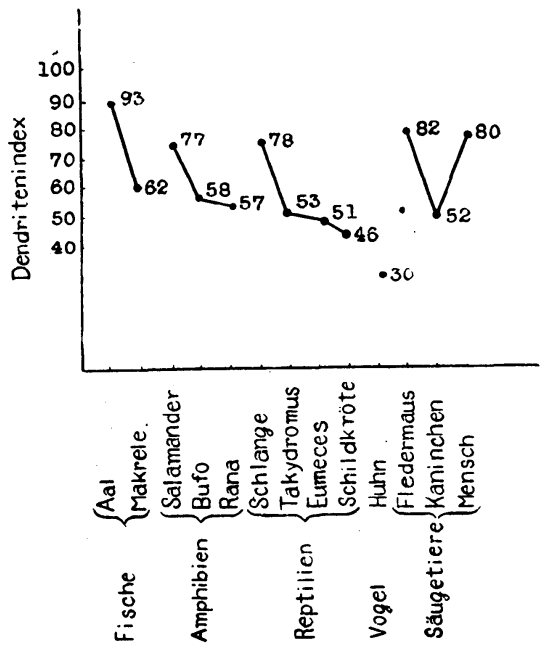

Abb. 3. Dendritenindex der bipolaren Zellen.

\section{Zusammenfassag.}

Die Untersuchungen wurden vorgenommen an den Fischen : Aal und Makrele, den Amphibien: Salamander, Bufo vulgaris und Rana nigromaculata, den Reptilien : Schlange, Takydromus tachydromoides, Eumeces latisculatus und Schildkröte, dem Vogel : Huhn und den Säugetieren : Fledermaus, Kaninchen und Mensch. Die Dicke der Netzhautschichten wurde genau gemessen.

1. Die relative Dicke der äußeren Körnerschicht (bezogen auf die ganze Retinadicke) ist bei den verschiedenen Tieren verschieden, und «war bei Fische=Säugetiere $>$ Amphibien $>$ Reptilien $>$ Vogel. Bei den Tieren, welche an den Stäbchenzellen reich sind, ist also dje relative Dicke groß, und bei denen, die an den Zapfenzellen reich sind, ist sie klein. Die relative Dicke der inneren Körnerschicht ist : Amphibien $>$ Reptilien $>$ Säugetiere $>$ Fische $>$ Vogel. Die relative Dicke der äußeren Körnerschicht ist bei den Fischen und Säugetieren größer als diejenige der inneren Körnerschicht, aber bei den Amphibien, Reptilien und dem Vogel findet sich das Umgekehrt.

2. Wenn bei den Fischen, Amphibien, Reptilien bzw. Säugetieren die relative Dicke der Zapfenstäbchenschicht (bezogen auf die ganze Retinadicke) groß ist, so ist auch diejenige der äußeren Körnerschicht 
groß. Auch bei jeder Tierart ist die relative Dicke der inneren Kör nerschicht umso kleiner, je größer der Augapfel ist. Die HENLEsche Faserschicht ist gut entwickelt bei den Tieren, welche im hellen wohnen. Die relative Dicke der Nervenfaserschicht ist groß bei solchen 'lieren, die eine gute Sehkraft haben.

3. Die relative Dicke der äußeren retikulären schicht und der Ganglienzellenschicht (bezogen auf die ganze Retinadicke) zeigt bei allen Tieren keine große Schwankung.

4. Bei den Fischen, Amphibien, Reptilien bzw. Säugetieren betätigte sich, daß bei jeder Tierart der Kernteil der bipolaren Zellen den Sehzellen näher steht, wenn das Tier im helleren wohnt. Es gilt also auch hier das KAPPERSsche Gesetz der Neurobiotaxis.

\section{內 容自 抄}

成長した魚類 (ウナギとサバ), 网嫩類 (キモリ, ヒキガヘル, .トノサマ ガへル), 伋虫類 (ヘビ, カナへビ, トカゲ, カメ), 鳥類(ニメトリ), 哺 乳類(カウモリ, 家鬼, 人)の眼球を固定後にチェロイヂン切片とし, 染色 したものにつき觀祭と計測が行はれた.

外顆粒愿の絧膜全愿に對する相對厚は魚類=哺乳類 $>$ 兩棲類 $>$ 鳥類の順

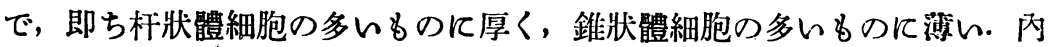
顆粒屏のものは, 兩棲類>爬虫類>哺乳類>魚類>鳥類の順である. 而し て外顆粒曆の相對厚は魚類と哺乳類では內顆粒層のものより大であるが, 兩棲類, 爬虫類, 鳥類では逆である. 魚類, 兩棲類, 爬虫類, 哺乳類の夫

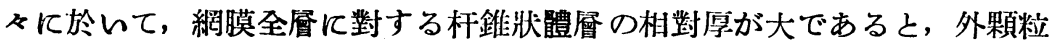

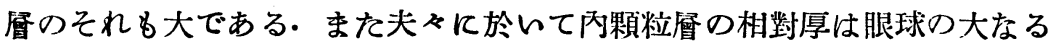

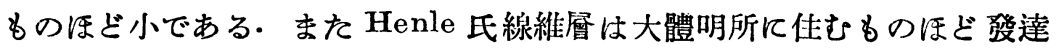

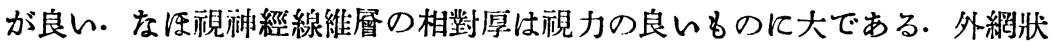
㕌之視神經節細胞展の網膜全愿に對する相對厚はすべての動物であまり變 らない.

魚類, 兩楼類, 吧虫類, 哺乳類の夫そに於んて, 絧膜の双極細胞の核部 分は明所に住むるのほど視細胞に近い. 即ち Kappers 氏神經生物制位の 法則に從つてみる.

\section{Literatur.}

Franz, v. P. : 1913. Aus Oppels Lehrbuch der vergl. mikrosk. Anatomie der Wirbeltiere. Bd. 7. 1913. - Kappers, C R. R. : On structural laws in the nervous system : The principles of neurobiotaxis. Brain 44 (1921). - Krause, W. : Die Retina. II. IV. VI. Internat. Mschr. Anat. Histol. 
10 (1893), 12 (1895). - Schultze, M. : Zur Anatomie und Physiologie der Retina. Arch. inikr. Anat. 2、1866). - Schultze, M. : Über Stäbchen und Zapfen der Retina. Arch. mikr. Anat. 3(1867). - Sudo, G. : Histometrische Studien über das Pigmentepithel und die Netzhaut des Huhnes. Okajimas Fol. anat. jap. 23 (1950),

\section{Erklärung der Tafelabbildungen.}

Abb. 1. Aal. Die äußere Körnerschicht ist dicker als die innere. Die HENLEsche Faserschicht fehlt.

Abb. 2. Makrele. Die HENLEsche Faserschicht ist gut entwickelt. Die äußere Körnerschicht ist dicker als die innere.

Abb. 3. Salamander. Die innere Körnerschicht ist dicker als die äußere. Die HENLEsche Faserschicht fehlt.

Abb. 4. Bufo vulgaris:

abb. 5. Rana nigromaculata.

Abb. 6. Schlange.

Abb. 7. Takydromus tachydromoides.

Abb. 8. Eumeces latisculatus.

Abb. 9. Schildkröte.

Abb. 10. Huhn. Die HENI.Esche Faserschicht ist entwickelt. Die innere Körnerschicht ist dicker, als die äußere.

Abb. 11. Fledermaus. Die äußere Körnerschicht ist dicker als die innere. Die HENLEsche Faserschicht ist nicht zu sehen.

Abb. 12. Kaninchen.

Abb. 13. Mensch. Die HENIEsche Faserschicht erscheint. Die äußere Körnerschicht ist dicker als die innere. 
須 藤 論 文 附 圖

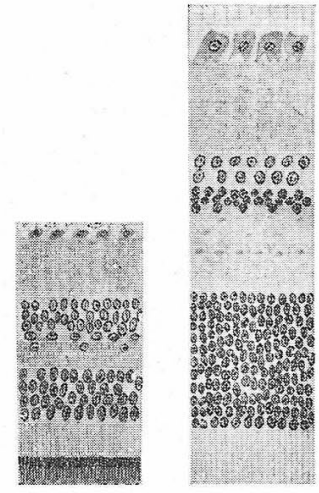

1.

Aal.
2.

Makrole.

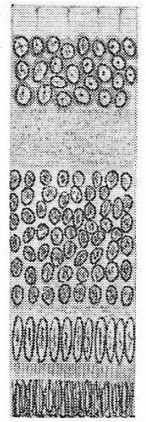

3.

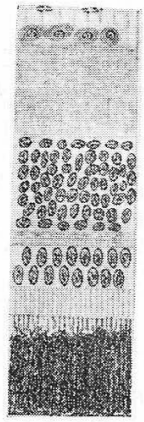

4.

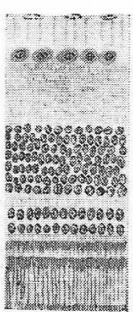

5.

Rana.

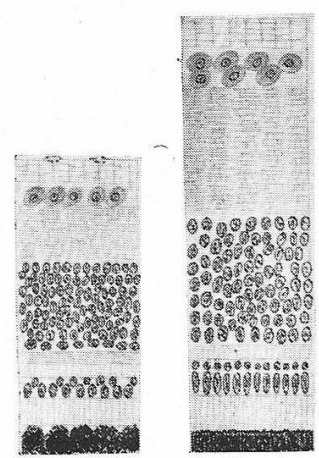

e.
7.

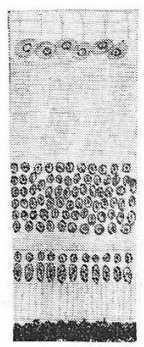

8.

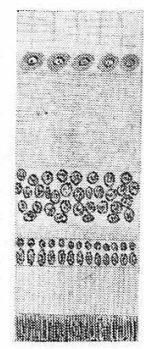

9.

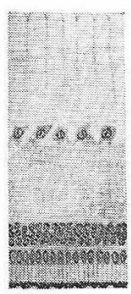

10.

Schlange. Takydromus, Eumeces. Schildkröte. Huhn.

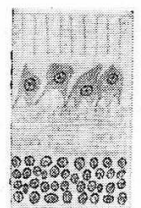

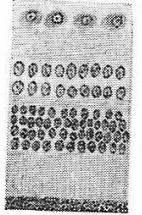

11.

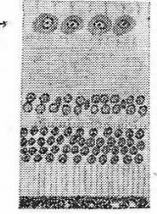

12.

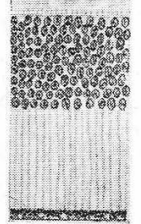

13.

Fledermaus. Kaninchen. Mensch. 